\title{
Mechanism of very high-energy radiation in BL Lacertae object 3C 66A (Research Note)
}

\author{
J. Yang ${ }^{1,2,3}$ and J. Wang ${ }^{1,2}$ \\ 1 National Astronomical Observatories, Yunnan Observatory, Chinese Academy of Sciences, Kunming 650011, PR China \\ e-mail: yangjp@ynao.ac.cn \\ 2 Key Laboratory for the Structure and Evolution of Celestial Objects, Chinese Academy of Sciences, Kunming 650011, PR China \\ 3 Yunnan Agricultural University, Kunming 650201, PR China
}

Received 31 July 2009 / Accepted 16 November 2009

\section{ABSTRACT}

\begin{abstract}
Aims. Our goal is to understand the nature of blazars and the mechanisms for generating high-energy $\gamma$-rays, through investigation of the blazar 3C 66A.

Methods. We model the high-energy spectrum of 3C 66A, which was observed recently with the Fermi-LAT and VERITAS telescopes. The spectrum has a hard change from the energy range of $0.2-100 \mathrm{GeV}$ to $200-500 \mathrm{GeV}$ in recent almost contemporaneous observations of two telescopes.

Results. The de-absorbed VERITAS spectrum depends strongly on redshift, which is highly uncertain. If $z=0.444$ is adopted, we are able to use the SSC model to produce the Fermi-LAT component and the EC model to the VERITAS component. However, if $z=0.1$, the intrinsic VERITAS spectrum will be softer, and there will be a smooth link between the Fermi-LAT and VERITAS spectra that can be explained using an SSC model.
\end{abstract}

Key words. BL Lacertae objects: individual: 3C 66A

\section{Introduction}

Blazars are a peculiar class of active galactic nuclei (AGN), and their jets point at small angles with respect to our line of sight. Many of them have been observed at all wavelengths, from radio to very high energy (VHE) $\gamma$-rays. Their spectral energy distribution (SED) consists of two bumps that are attributed to the synchrotron and the inverse Compton (IC) emission of ultrarelativistic particles. The different soft-photon sources deduce synchrotron self-Compton (SSC) and external Compton (EC) models to produce high-energy emission. In the SSC model, the soft photons are provided by the synchrotron emission of the same electrons (Marscher 1980; Ghisellini \& Maraschi 1989; Marscher \& Travis 1996); however, in the EC model, the soft photons mostly come from the outside of the jet, such as outer disk, broad-line region (BLR) clouds (etc. Dermer et al. 1993; Sikora et al. 1994).

In 3C 66A, Miller et al. (1978) give the redshift $z=0.444$ by a weak $\mathrm{Mg}$ II emission line detection, but it is very uncertain (Bramel et al. 2005). When 3C 66A is located at $z=0.444$, its $\mathrm{TeV}$ photons will suffer from the strong pair production absorption of the extragalactic background light (EBL). After correction by the EBL absorption, TeV emission presents an inverted intrinsic spectrum (see the Acciari et al. 2009. Figure 2, the deabsorbed photon spectral index is calculated as $1.1 \pm 0.4)$. In this paper, we take $z=0.444$ to analyze TeV emissive mechanism and discuss the behavior of the de-absorbed VERITAS spectrum in different redshifts. Generally, 3C 66A is classified as a low-frequency peaked BL Lac object (LBL). The peak of the low-frequency component of LBLs usually lies in the IR or optical regime, whereas the peak of high-energy component is located at several GeV. The luminosity of $\gamma$-ray is typically comparable to or slightly higher than the luminosity of the synchrotron component. Such as, Joshi \& Bottcher (2007) argue that the peak of the low-frequency component for $3 \mathrm{C} 66 \mathrm{~A}$ is located at the optical regime, and the peak of high-frequency component reaches a multi $\mathrm{MeV}-\mathrm{GeV}$ range. However, Perri et al. (2003) reveal that the synchrotron peak is found between $10^{15}$ and $10^{16} \mathrm{~Hz}$, then $3 \mathrm{C} 66 \mathrm{~A}$ is classified as an intermediatefrequency peaked BL Lac (IBL). From the X-ray spectrum with the photon spectral index $\Gamma \sim 2.5$ (Bottcher et al. 2005; Donato et al. 2005; Foschini et al. 2006), which might be the tail of the synchrotron emission, $3 \mathrm{C} 66 \mathrm{~A}$ is considered as an IBL in this paper. 3C 66A is observed in radio, IR, optical, X-rays, and $\gamma$-rays and shows strong luminosity variations. As described in Bottcher et al. (2005), the object exhibits several outbursts in the optical band and the variations of $\Delta m \sim 0.3-0.5$ over several days. Until now, the majority of BL Lacs detected at VHE (very high energy: $E>100 \mathrm{GeV}$ ) are HBLs (high-frequency peaked BL Lacs). Only IBL W Comae (Acciari et al. 2008), LBL BL Lacertae (Albert et al. 2007), and 3C 279 (Albert et al. 2008) display the potential to enlarge the extragalactic $\mathrm{TeV}$ source. For 3C 66A, the Crimean Astrophysical Observatory report a 5.1 $\sigma$ detection above $900 \mathrm{GeV}$ (Stepanyan et al. 2002).

Recently, VERITAS have carried out $14 \mathrm{~h}$ of observations for 3C 66A from September 2007 through January 2008 (hereafter, the 2007-2008 season) and from September through November 2008 (hereafter, the 2008-2009 season), a further $46 \mathrm{~h}$ of data were taken (Acciari et al. 2009). Because of the limited spatial resolution of Cherenkov telescopes, it is difficult to accurately 
identify the emission region. The radio galaxy $3 \mathrm{C} 66 \mathrm{~B}$ lies in the same view field of $3 \mathrm{C} 66 \mathrm{~A}$ at a separation of $0.12^{\circ}$ and is also a plausible source of VHE radiation (Tavecchio \& Ghisellini 2008). The recent detection by MAGIC favored $3 \mathrm{C} 66 \mathrm{~B}$ as VHE source and excluded $3 \mathrm{C} 66 \mathrm{~A}$ at an $85 \%$ confidence level (Aliu et al. 2009). However, VERITAS have found that $3 \mathrm{C}$ 66A lies $0.01^{\circ}$ from the fit position, while $3 \mathrm{C} 66 \mathrm{~B}$ lies $0.13^{\circ}$ away, and $3 \mathrm{C} 66 \mathrm{~A}$ is VHE source. If $3 \mathrm{C} 66 \mathrm{~A}$ has a redshift of $z=0.444$, its de-absorbed spectral index is $1.1 \pm 0.4$ showing very hard intrinsic spectrum (Acciari et al. 2009). In the first three months, the Fermi-LAT Gamma-ray Space Telescope have observed 3C 66A (Abdo et al. 2009), almost at contemporaneous observation with VERITAS in the 2008-2009 season. However, very soft spectrum with the spectral index of $1.97 \pm 0.04$ appears in the Fermi-LAT observing energy range. The $\gamma$-ray spectrum suddenly hardens from $0.2-100 \mathrm{GeV}$ to $200-500 \mathrm{GeV}$ and challenges the one-zone homogeneous SSC model.

In Sect. 2 we present the jet models for application to $3 \mathrm{C}$ 66A. We use the observed data to constrain the model parameters in Sect. 3. We finish with discussions and conclusions in Sect. 4. Throughout this paper, we use a soft cosmology with a deceleration factor $q_{0}=0.5$ and a Hubble constant $H_{0}=$ $75 \mathrm{~km} \mathrm{~s}^{-1} \mathrm{Mpc}^{-1}$.

\section{The models}

We use a public model of Georganopoulos et al. (2007) ${ }^{1}$ to describe the observed spectrum of $3 \mathrm{C} 66 \mathrm{~A}$. The emission region is assumed to be a sphere (blob) with radius $R$, permeated by a homogeneous magnetic field $B$. The blob moves with bulk Lorentz factor $\Gamma$ through an external photon field with a black body spectrum, at an angle $\theta$ (in this work $\theta \sim 1 / \Gamma$ is assumed) with respect to the line of sight, and has a Doppler factor $\delta \sim \Gamma$. The relativistic electrons are continuously injected into the blob at a rate $Q_{\mathrm{e}}(\gamma)=Q_{0} \gamma^{-s}\left[\mathrm{~cm}^{-3} \mathrm{~s}^{-1}\right]$ between $\gamma_{\min }$ and $\gamma_{\max }$, where $\gamma_{\min }$ is the minimum Lorentz factor of the injected electrons and should not be confused with the minimum Lorentz factor of the emitting particles. The injection correspond to a luminosity $L_{\mathrm{inj}}$,

$$
Q_{0}= \begin{cases}\frac{L_{\mathrm{inj}}(2-s)}{V m_{\mathrm{e}} c^{2}\left(\gamma_{\max }^{2-s}-\gamma_{\min }^{2-s}\right)}, & s \neq 2, \\ \frac{L_{\mathrm{inj}}}{V m_{\mathrm{e}} c^{2} \ln \frac{\gamma_{\max }}{\gamma_{\min }}}, & s=2 .\end{cases}
$$

Where $V=4 \pi R^{3} / 3$ is the volume of the blob. Injected electrons might obtain energy from acceleration processes before into the emission region, such as diffusive shock acceleration, second order Fermi acceleration and gradual shear acceleration. Detailed discussions about the acceleration processes are beyond the scope of this paper. The electrons suffer synchrotron and inverse Compton losses and eventually escape from the emission region.

The time-dependent evolution of the electron population $N(\gamma, t)\left[\mathrm{cm}^{-3}\right]$ inside the emission region is governed by

$\frac{\partial N(\gamma, t)}{\partial t}=-\frac{\partial}{\partial \gamma}\left[\left(\frac{\mathrm{d} \gamma}{\mathrm{d} t}\right)_{\mathrm{loss}} N(\gamma, t)\right]+Q_{\mathrm{e}}(\gamma)-\frac{N(\gamma, t)}{t_{\mathrm{esc}}}$

Here, $(\mathrm{d} \gamma / \mathrm{d} t)_{\text {loss }}$ is the radiative energy loss rate, because of synchrotron, SSC, and/or EC emission. The electron escape

\footnotetext{
1 Georganopoulos, M., Kazanas D., Perlman, E., et al. 2007, http://jca. umbc . edu/ markos/cs/index . html
}

timescale, $t_{\mathrm{esc}}$, which is several light crossing times, in this work $5 R / c$ is adopted.

The energy loss rates of electrons caused by the synchrotron are given by $\dot{\gamma}_{\mathrm{s}}=\frac{4 \sigma_{\mathrm{T}}}{3 m_{\mathrm{c}} c} \gamma^{2} U_{B}$, where $U_{B}=B^{2} /(8 \pi)$ is the magnetic energy density. The energy loss rates of inverse Compton emission, $\dot{\gamma}_{\mathrm{IC}}$, have excellent analytical expressions for the Thomson regime $x \ll 1$ and for the deep Klein-Nishina (KN) regime $x \gg 1$, but do not have ones for the middle regime of $x=\epsilon \gamma$ (where $\epsilon$ is the energy of the incoming photon in units of the electron rest mass). To overcome this, Georganopoulos et al. (2007) modifies an analytical approximation used by Moderski et al. (2005): $\dot{\gamma}_{\mathrm{IC}}=\frac{4 \sigma_{\mathrm{T}}}{3 m_{\mathrm{e}} c} \gamma^{2} U_{\mathrm{r}} F_{\mathrm{KN}}$, where $U_{\mathrm{r}}$ is the energy density of seed photons including synchrotron photons and external photons (such as the reprocessed photons by broad line region (BLR) Sikora et al. 1994), $F_{\mathrm{KN}}$ is given by Moderski et al. (2005). In this paper, the latter photons are assumed to be a blackbody radiation with peak frequency $v_{\mathrm{ext}}$ and energy density $U_{\text {ext }}$ (all seen in the observer frame). For the beaming of the EC emission, we use the recipe of Georganopoulos et al. (2001).

\section{Modeling parameters}

First of all, we use the observed quantities to estimate the physical parameters in the blob, and then use these values to reproduce the observed SED. Mastichiadis \& Kirk (1997) and Konopelko et al. (2003) have estimated the parameter relations of the inverse Compton scattering in the Klein-Nishina regime in a homogeneous SSC scenario. However, Paggi et al. (2009) find that the simple relations of parameters in Thomson regime satisfy the observations of LBL or IBL sources and that the relations in the extreme KN limit are not suited to HBLs, indicating that the inverse Compton scattering for HBLs just borders the KN regime. For 3C 66A, the Fermi-LAT spectra showing flat and rising shapes and higher energy observations of VERITAS both indicate that the IC scattering of Fermi-LAT spectra are not likely to enter into the Klein-Nishina regime. Therefore, we assume that the Fermi-LAT spectrum just covers the peak of SSC emission, so use the parameter relations in Thomson regime to rudely estimate the parameters. In fact, we consider the Klein-Nishina effect in producing the high-energy spectra.

The size of the emitting region, $R$, can be constrained by measurements of variability time scales, i.e. $R \leq \delta c t_{\mathrm{var}} /(1+z)$. The observations of VERITAS have shown the variability to be on a time scale of days (Acciari et al. 2009). The multiwave campaign of 3C 66A by the Whole Earth Blazar Telescope (WEBT) in 2007-2008 has observed several bright flares on time scales of 10 days (Bottcher et al. 2005). These observations suggest that the size of the emitting region is about $10^{16} \mathrm{~cm}$ if we assume $\delta$ is close to 10 . From the peak frequencies of synchrotron and inverse Compton radiation, we can estimate the $\gamma_{\text {peak }}$ (i.e. the Lorentz factor of the electrons emitting at the peaks of the synchrotron and SSC components) as (Tavecchio et al. 1998)

$v_{s}=\frac{4}{3} v_{L} \gamma_{\text {peak }}^{2} \delta /(1+z)$

$v_{\mathrm{SSC}} \simeq \frac{4}{3} v_{s} \gamma_{\text {peak }}^{2}$

and we obtain

$$
\gamma_{\text {peak }} \simeq\left(\frac{3 v_{\mathrm{SSC}}}{4 v_{s}}\right)^{1 / 2}
$$


Table 1. Parameters for the SSC+EC or SSC model used to reproduce the SED (Figs. 1 and 3).

\begin{tabular}{lll}
\hline \hline Parameters & If $z=0.444$ & If $z=0.1$ \\
\hline$L_{\text {inj }}\left(10^{41} \mathrm{erg} \mathrm{s}^{-1}\right)$ & 4.0 & 6.3 \\
$\gamma_{\min }$ & $2.5 \times 10^{4}$ & $1.5 \times 10^{4}$ \\
$\gamma_{\max }$ & $8.0 \times 10^{5}$ & $8.0 \times 10^{5}$ \\
$s$ & 3.4 & 3.3 \\
$R\left(10^{16} \mathrm{~cm}\right)$ & 7.0 & 7.0 \\
$\delta$ & 35 & 30 \\
$B(\mathrm{Gauss})$ & 0.034 & 0.05 \\
$v_{\mathrm{ext}}(\mathrm{Hz})$ & $2.5 \times 10^{15}$ & -- \\
$U_{\text {ext }}\left(\mathrm{erg} / \mathrm{cm}^{3}\right)$ & $3.5 \times 10^{-6}$ & -- \\
\hline
\end{tabular}

and

$\delta B \simeq(1+z) \frac{v_{s}^{2}}{v_{\mathrm{SSC}} \cdot\left(e / 2 \pi m_{\mathrm{e}} c\right)}$,

where $v_{L}=e B / 2 \pi m_{\mathrm{e}} c \simeq 2.8 \times 10^{6} \mathrm{~B} \mathrm{~Hz}$. From the equation

$\frac{L_{\mathrm{SSC}}}{L_{s}}=\frac{L_{\mathrm{SSC}}^{\prime}}{L^{\prime}{ }_{s}}=\frac{\dot{\gamma}_{\text {peak }, \mathrm{SSC}}}{\dot{\gamma}_{\text {peak }, \mathrm{s}}} \simeq \frac{U^{\prime}{ }_{s}}{U^{\prime}{ }_{B}} \simeq \frac{L_{s}}{\delta^{4} 4 \pi R^{2} c} \frac{8 \pi}{B^{2}}=\frac{2 L_{s}}{\delta^{4} R^{2} B^{2} c}$

we have

$\delta^{4} B^{2} \simeq \frac{2 L_{s}^{2}}{L_{\mathrm{SSC}} R^{2} c} \simeq \frac{8 \pi d_{l}^{2} \cdot\left(v_{s} F_{\nu, s}\right)^{2}}{\left(v_{\mathrm{SSC}} F_{\nu, \mathrm{SSC}}\right) \cdot R^{2} c}$,

where $L_{s}$ and $L_{\mathrm{SSC}}$ are the observed total luminosity of the synchrotron peak and SSC peak, $L_{\mathrm{SSC}}^{\prime}$ and $L^{\prime}{ }_{s}$ are the luminosity in the comoving frame, $\dot{\gamma}_{\text {peak,s }}$ and $\dot{\gamma}_{\text {peak,SSC }}$ denote the synchrotron and SSC cooling rates of the electrons, $U^{\prime}{ }_{S}$ and $U^{\prime}{ }_{B}$ are the comoving energy densities of synchrotron photons and magnetic fields, $d_{l}$ is the luminosity distance, and $F_{v, s}$ and $F_{v, \text { SSC }}$ are the energy flux at peaks. Using Eqs. (6) and (8) and $R \approx \delta c t_{\mathrm{var}} /(1+z)$ (here, $t_{\mathrm{var}} \approx 2$ days is assumed), we are able to derive the $\delta$ and $B$ from $v_{s}, v_{\mathrm{SSC}}, v_{s} F_{v, s}$, and $v_{\mathrm{SSC}} F_{v, \mathrm{SSC}}$. We take $v_{s} \approx 4 \times 10^{15} \mathrm{~Hz}$ (Perri et al. 2003), $v_{s} F_{v, s} \approx 7 \times 10^{-11} \mathrm{erg} \mathrm{cm}^{-2} \mathrm{~s}^{-1}$ estimated from historical data, $v_{\mathrm{SSC}} \approx 1.21 \times 10^{25} \mathrm{~Hz}$, which is the middle band of Fermi-LAT, $v_{\mathrm{SSC}} F_{v, \mathrm{SSC}} \approx 4.9 \times 10^{-11} \mathrm{erg} \mathrm{cm}^{-2} \mathrm{~s}^{-1}$ which is an approximative value observed by the Fermi-LAT, and $z=0.444$. We then get $\delta \sim 29$ and $B \sim 0.025 \mathrm{G}$. Also, $\delta$ can be estimated by other methods. The apparent speed $\beta_{\text {app }}=12.1 \mathrm{c}$ is presented by Bottcher et al. (2005). Modeling the non-simultaneous SED of 3C 66A, Ghisellini et al. (1998) suggest $\Gamma \sim 14$, which is a typical value for blazars. Jorstad et al. (2001) favor a high superluminal speed up to $\beta_{\text {app }} \approx 27 c$ and get $\Gamma \geq 27$ or $\delta \sim 30$. However, it is noted that we use the flux of the Fermi-LAT band to estimate the parameters. If the modeling spectrum includes the VERITAS spectrum, the estimated parameters will be modified. In fact, modeling quasi-simultaneous observations of Fermi-LAT and VERITAS leads to $\delta=35$ (see Table 1.).

The steady state distribution of emitting electrons is given by

$N(\gamma)=\frac{\int\left[Q_{\mathrm{e}}(\gamma)-N(\gamma) / t_{\mathrm{esc}}\right] \mathrm{d} \gamma}{\dot{\gamma}}$.

What is the criterion for steady state? The code of Georganopoulos et al. (2007) first takes a time step equal to the cooling time of the lowest energy electrons under the synchrotron and external Compton losses, and then calculates $N(\gamma)$ to a steady state where the injected electrons have fully been cooled, through an adaptive time step.

We emphasize the selection of the spectral index $s$ of injected electrons. Based on the X-ray photon spectral index of $\Gamma \approx 2.5$ (Bottcher et al. 2005; Donato et al. 2005; Foschini et al. 2006), we deduce the spectral index of emitting electrons to be 4 . Assuming the observed X-rays to be from synchrotron emission of the cooled electrons, the spectral index of the injected electrons is given by $s=3$. Celotti \& Ghisellini (2008) adopt $s=3.6$ to model the SED of 3C 66A. Therefore, we use $s \sim 3$ to model the observed data. The particle injection, radiative cooling, and escape from the emission region might yield a temporary quasi-equilibrium state described by a broken power law. The balance between escape and radiative cooling will lead to a break in the equilibrium particle distribution at a break Lorentz factor $\gamma_{\mathrm{b}}$, where $t_{\mathrm{esc}}=t_{\mathrm{cool}}\left(\gamma_{\mathrm{b}}\right)$. The cooling time scale is evaluated taking synchrotron, SSC, and EC cooling into account. Depending on whether $\gamma_{\mathrm{b}}$ is greater than or less than $\gamma_{\min }$, the system will be in the slow-cooling or fast-cooling regime. In the fast-cooling regime $\left(\gamma_{\mathrm{b}}<\gamma_{\min }\right)$, the equilibrium distribution will be a broken power law with $N(\gamma) \propto \gamma^{-2}$ for $\gamma_{\mathrm{b}}<\gamma<\gamma_{\min }$ and $N(\gamma) \propto \gamma^{-(s+1)}$ for $\gamma_{\min }<\gamma<\gamma_{\max }$. In the slow-cooling regime $\left(\gamma_{\mathrm{b}}>\gamma_{\min }\right)$, the equilibrium distribution will be $N(\gamma) \propto \gamma^{-2}$ for $\gamma<\gamma_{\min }, N(\gamma) \propto \gamma^{-s}$ for $\gamma_{\min }<\gamma<\gamma_{\mathrm{b}}$, and $N(\gamma) \propto \gamma^{-(s+1)}$ for $\gamma_{\mathrm{b}}<\gamma<\gamma_{\max }$. Since for thin synchrotron emission, the energy spectral index is related to that of the emitting electrons as $\alpha=(q-1) / 2$, where $q$ is the spectral index of the emitting electron distribution, and can immediately be seen that the peak in the $v F_{v}$ spectrum occurs where $q=3$. When $s>3$ (in this work $s=3.4$ and 3.3 are adopted), $\gamma_{\min }$ (the minimum Lorentz factor of the injected electrons) almost corresponds to $\gamma_{\text {peak }}$ (Ghisellini et al. 1998, 2002). Another input parameter, $\gamma_{\max }$, presents the balance between the acceleration and cooling and has a low impact upon the SED. It is usually taken to be $10^{5}-10^{7}$ (Inoue \& Takahara 1996).

Considering the EC emission of electrons, we need to estimate $U_{\text {ext }}$ and $v_{\text {ext }}$ by mainly considering the soft photons reprocessed by the BLR. Usually $v_{\mathrm{ext}}$ is considered to be around optical-UV wavebands, so we let $v_{\mathrm{ext}}=2.5 \times 10^{15} \mathrm{~Hz}$. The $L_{\text {ext }}$ of FSRQ is easily estimated from emission line spectra or UV-excesses, while it is difficult to estimate the $L_{\text {ext }}$ of BL Lacs. Assuming the luminosity of the accretion disk to be $L_{\mathrm{D}}=$ $1 \times 10^{45} \mathrm{erg} \mathrm{s}^{-1}$, which is more than the jet luminosity and does not produce a blue bump in the simulated SED (Joshi \& Bottcher 2007). Taking $R_{\text {ext }} \sim 10^{17} \mathrm{~cm}$ (Tavecchio \& Ghisellini 2008), we get the upper limit $U_{\text {ext, }}=2.65 \times 10^{-2} \mathrm{erg} \mathrm{cm}^{-3}$, assuming the reprocessing efficiency of the BLR to be 0.1 . In this work, we estimate $U_{\text {ext }}$ through modeling the spectrum under the condition of $U_{\text {ext }} \leq U_{\text {ext,u }}$. Taking $U_{\text {ext }}=3.5 \times 10^{-6} \mathrm{erg} \mathrm{cm}^{-3}$, we can already reproduce the observed data of VERITAS. Therefore the soft photons reprocessed by the BLR can provide the EC emission of electrons to produce high-energy radiation.

In Fig. 1, we present the modeling results for the observed data of 3C 66A, which assemble the data of Perri et al. (2003), Bottcher et al. (2009), Fermi-LAT, and the VERITAS results corrected by EBL absorption according to Franceschini et al. (2008). We include a quasi-simultaneous data including the nearinfrared, optical, UV-Optical, and X-ray observations (Reyes et al. 2009). We used the synchrotron emission model to model the lower energy part, and used the SSC model to reproduce the spectrum observed by the Fermi-LAT. Particularly we use the EC model to model the VERITAS spectrum. It is indicated that the harden spectrum from the Fermi-LAT to VERITAS energy ranges could exhibit an EC spectrum. 


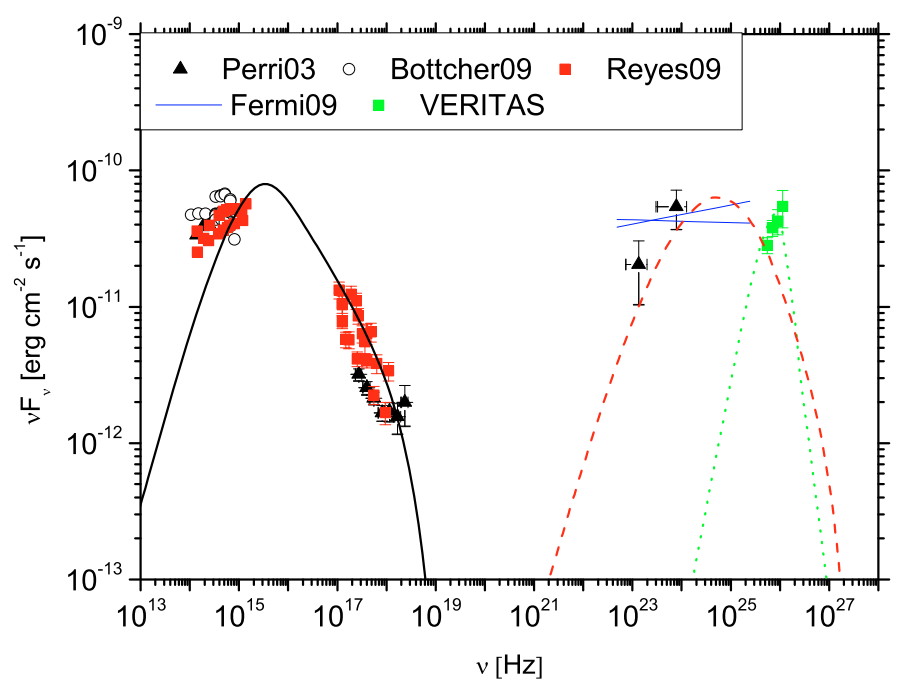

Fig. 1. Modeling SED of 3C 66A with the SSC+EC model. The black triangles are the data of Perri et al. (2003), the open circles come from Bottcher et al. (2009), the cross blue bow-tie show the Fermi-LAT spectrum, the red squares denote the data of Reyes et al. (2009), and the green squares represent the VERITAS spectrum corrected by EBL absorption according to Franceschini et al. (2008). We use the synchrotron model to model the lower energy part (black solid line), the SSC model to reproduce the Fermi-LAT spectrum (red dash line), and the EC model to model the VERITAS spectrum (green dot line).

\section{Discussions and conclusions}

The redshift of 3C 66A has an uncertain value (Bramel et al. 2005), and is usually adopted as $z=0.444$; however, the redshift is crucial in constructing intrinsic high-energy spectrum because of the EBL absorption (Hauser \& Dwek 2001). This absorption decreases the observed flux and softens the observed spectrum. If the redshift is less than 0.444 , such as just $\geq 0.096$ suggested by Finke et al. (2008), the intrinsic spectrum in the VERITAS energy range will be softer. There will be a smooth link spectrum between the Fermi-LAT and the VERITAS energy ranges. In Fig. 2, we generate the intrinsic spectra of VERITAS observations corrected by EBL absorption according to Franceschini et al. (2008) model, assuming the source to be at the different redshifts $z=0.03,0.1,0.3$, and 0.5 . It is shown that the de-absorbed spectrum strongly depends on the redshift. When $z=0.3$, the de-absorbed spectrum has a little inverted, but it becomes an inverted spectrum in $z=0.5$. If the redshift is less than 0.1 , the de-absorbed spectrum will present the usual SED of an LBL such as W Comae (Acciari et al. 2008). In Fig. 3, we show the de-absorbed SEDs under $z=0.1$ and the modeling. A smooth spectrum can link the Fermi-LAT and VERITAS data and be explained with an SSC model, in which $U_{B}=9.95 \times 10^{-5} \mathrm{erg} \mathrm{cm}^{-3}$ and $U_{\mathrm{e}}=2.31 \times 10^{-3} \mathrm{erg} \mathrm{cm}^{-3}$.

In fact the bulk motion of the emitting blob affects the observed SED (e.g., Dermer 1995; Georganopoulos et al. 2001). The peak frequencies are given by $v_{s} \approx 3.7 \times 10^{6} \gamma_{\text {peak }}^{2} \delta B /(1+z)$, $v_{\mathrm{SSC}} \approx \frac{4}{3} \gamma_{\text {peak }}^{2} v_{s}$, and $v_{\mathrm{EC}} \approx \frac{4}{3} \gamma_{\text {peak }}^{2} \nu_{\mathrm{ext}} \Gamma \delta /(1+z)$. We can see that $\nu_{\mathrm{EC}}$ would be higher than $v_{\mathrm{SSC}}$ provided the blob has larger $\delta$ or $\Gamma$ (Usually the viewing angle $\theta \sim 1 / \Gamma$ is assumed, $\delta \sim \Gamma$.) From the ratio of peak luminosity, $\frac{L_{\mathrm{EC}}}{L_{\mathrm{SSC}}} \approx \frac{L_{\mathrm{EC}}}{L_{\mathrm{SSC}}}=\frac{\dot{\gamma}_{\text {peak }, \mathrm{EC}}}{\dot{\gamma}_{\text {peak }, \mathrm{SSC}}} \approx \frac{U^{\prime}{ }_{\mathrm{xxt}}}{U_{\text {syn }}} \approx$ $\delta^{4} \Gamma^{2} \frac{\xi L_{\text {ext }}}{L_{s}} \frac{R^{2}}{R_{\text {ext }}^{2}}\left(U_{\text {ext }}\right.$ is amplified by a factor of $\Gamma^{2}$, see Sikora et al. 1994; and Dermer 1995), where $\xi$ is the reproduced fraction of the $L_{\text {ext }}$, we show that $\frac{L_{\mathrm{EC}}}{L_{\mathrm{SSC}}}$ is strongly affected by the bulk

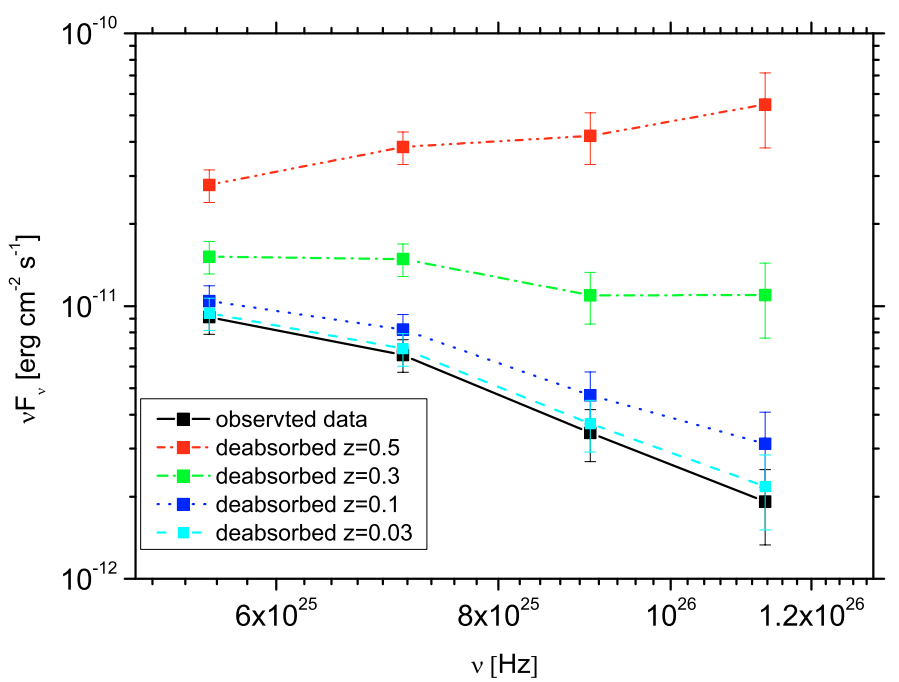

Fig. 2. Intrinsic spectra of VERITAS observations corrected by EBL absorption according to the Franceschini et al. (2008) model in different redshifts, such as $z=0.03$ (cyan dash line), 0.1 (blue dot line), 0.3 (green dash-dot line), 0.5 (red dash-dot-dot line).

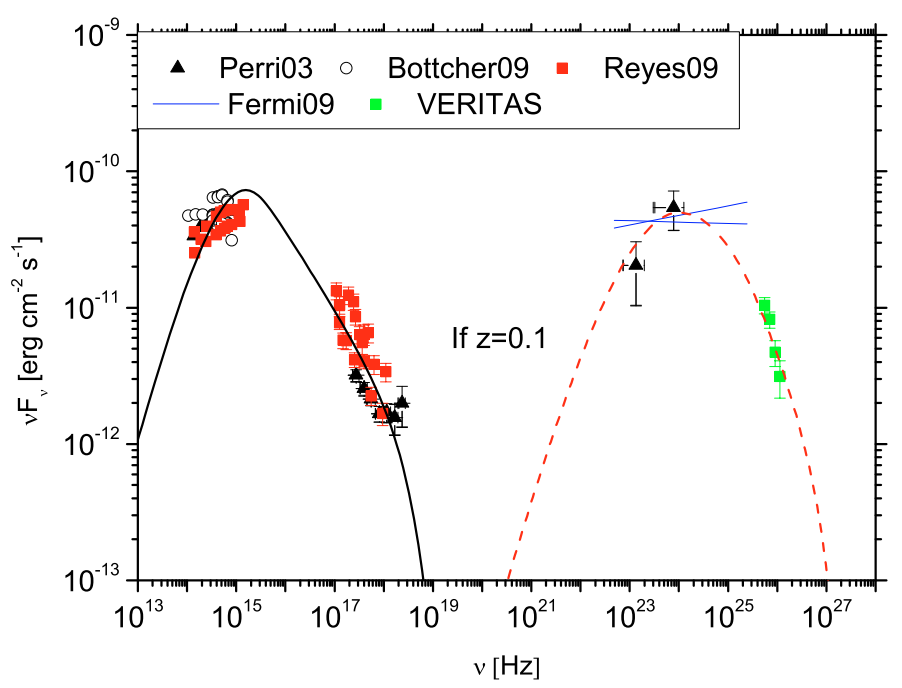

Fig. 3. Modeling SED of 3C 66A with the SSC model. The black triangles are the data of Perri et al. (2003), the open circles come from Bottcher et al. (2009), the cross blue bow-tie show the Fermi-LAT spectrum, the red squares present the data of Reyes et al. (2009), and the green squares denote the VERITAS spectrum corrected by EBL absorption according to Franceschini et al. (2008). We use the synchrotron model to model the lower energy part (black solid line) and the SSC model to reproduce the Fermi-LAT and de-absorbed VERITAS spectra assuming $z=0.1$ (red dash line).

motion of the blob. In Fig. 1, using $U^{\prime}{ }_{B}=4.6 \times 10^{-5} \mathrm{erg} \mathrm{cm}^{-3}$, $U_{\mathrm{e}}^{\prime}=1.67 \times 10^{-3} \mathrm{erg} \mathrm{cm}^{-3}$, and $U_{\text {ext }}^{\prime}=3.5 \times 10^{-6} \mathrm{erg} \mathrm{cm}^{-3}$, we can model the SED. $U_{\text {ext }}^{\prime}$ is obviously lower than $U_{B}^{\prime}$; however, the EC luminosity is comparable with the synchrotron ones (see Fig. 1). In the observer frame, the beaming factor is different for EC ( $\delta^{4+2 \alpha}$ Dermer 1995; Georganopoulos et al. 2001), synchrotron and SSC emission $\left(\delta^{3+\alpha}\right)$. The difference of EC and synchrotron luminosity is reasonable by considering their beaming factor.

The EC emission is less clear for the BL Lac objects. The lack of strong emission lines and UV excesses suggests that the external photon density $\xi L_{\text {ext }}$ is very low, while the Lorentz factor of BL Lac objects is typically less than that of quasars 
(Piner et al. 2008). Their high-energy emission strongly favors the SSC mechanism over the EC mechanism. But, 3C 66A might be an exception and reveal higher bulk velocity in the highenergy emissive region. Therefore, the high-energy emission caused by EC mechanism is likely observed in the IBL. This possibility needs future Fermi-LAT and VERTAS observations for $3 \mathrm{C} 66 \mathrm{~A}$ and a precise redshift determination.

Acknowledgements. We thank the referee for a very helpful and constructive report that helped to improve our manuscript substantially. We acknowledge the financial supports from the National Natural Science Foundation of China 10673028 and 10778702, and the National Basic Research Program of China (973 Program 2009CB824800).

\section{References}

Abdo, A. A., et al. 2009, ApJ, 701, 597

Acciari, V. A., Aliu, E., Beilicke, M., et al. 2008, ApJ, 684, L73

Acciari, V. A., Aliu, E., Arlen, T., et al. 2009, ApJ, 693, L104

Albert, J., Aliu, E., Anderhub, H., et al. 2007, ApJ, 666, L17

Albert, J., Aliu, E., Anderhub, H., et al. 2008, Science, 320, 1752

Albert, J., Anderhub, H., Antonelli, L. A., et al. 2009, ApJ, 692, L29

Bottcher, M., Harvey, J., Joshi, M., et al. 2005, ApJ, 631, 169

Bottcher, M., Fultz, K., Aller, H. D., et al. 2009, ApJ, 694, 174

Bramel, D. A., Carson, J., Covault, C. E., et al. 2005, ApJ, 629, 108

Celotti. A., \& Ghisellini, G. 2008, MNRAS, 385, 283

Dermer, C. D. 1995, ApJ, 446, L63

Dermer, C. D., \& Schlickeiser, R. 1993, ApJ, 416, 458

Donato, D., Sambruna, R. M., \& Gliozzi1, M. 2005, A\&A, 433, 1163
Finke, J. D., Shields, J. C., Böttcher, M., \& Basu, S. 2008, A\&A, 477, 513

Foschini, L., Ghisellini, G., Raiteri, C. M., et al. 2006, A\&A, 453, 829

Franceschini, A., Rodighiero, G., \& Vaccari, M. 2008, A\&A, 487, 837

Georganopoulos, M., \& Kazanas, D. 2003, ApJ, 594, L27

Georganopoulos, M., Kirk, J. G., \& Mastichiadis, A. 2001, ApJ, 561, 111

Ghisellini, G., \& Maraschi, L. 1989, ApJ, 340, 181

Ghisellini, G., Celotti, A., Fossati, G., Maraschi, L., \& Comastri, A. 1998, MNRAS, 301, 451

Ghisellini, G., Celotti, A., \& Costamante, L. 2002, A\&A, 386, 833

Hauser, M. G., \& Dwek, E. 2001, ARA\&A, 39, 249

Jorstad, S. G., Marscher, A. P., Mattox, J. R., et al. 2001, ApJS, 134, 181

Joshi, M., \& Bottcher, M. 2007, ApJ, 662, 884J

Kirk, J. G., Rieger, F. M., \& Mastichiadis, A. 1998, A\&A, 333, 452

Konopelko, A., Mastichiadis, A., Kirk, J., de Jager, O. C., \& Stecker, F. W. 2003, ApJ, 597, 851

Marscher, A. P. 1980, ApJ, 235, 386

Marscher, A. P., \& Travis, J. P. 1996, A\&AS, 120, 537

Mastichiadis, A., \& Kirk, J. 1997, A\&A, 320, 19

Miller, J. S., French, H. B., \& Hawley, S. A. 1978, in Pittsburgh Conference on BL Lac Objects, ed. A. M. Wolfe (Pittsburgh: Univ. Pittsburgh), 176

Moderski, Sikora, M., Coppi, P. S., \& Aharonian, F. 2005, MNNRAS, 363, 954

Paggi, A., Massaro, F., Vittorini, V., et al. 2009, A\&A, 504, 821

Perri, M., Massaro, E., Giommi, P., et al. 2003, A\&A, 407, 453

Piner, B. G., Pant, N., \& Edwards, P. G. 2008, ApJ, 678, 64

Reyes, L. C., et al. 2009, in The Proceedings of the 31st International Cosmic Ray Conference, [arXiv: astro-ph/0907.5175]

Sikora, M., Begelman, M. C., \& Rees, M. J. 1994, ApJ, 421, 153

Stepanyan, A. A., Neshpor, Yu. I., Andreeva, N. A., et al. 2002, Astron. Rep., 46,634

Tavecchio, F., Maraschi, L., \& Ghisellini, G. 1998, ApJ, 509, 608

Tavecchio, F., \& Ghisellini, G. 2008, MNRAS, 386, 945 